\title{
A simulation pipeline for the Planck mission $\star$
}

\author{
M. Reinecke ${ }^{1}$, K. Dolag ${ }^{1}$, R. Hell ${ }^{1}$, M. Bartelmann ${ }^{2,1}$, and T. A. Enßlin ${ }^{1}$ \\ 1 Max-Planck-Institut für Astrophysik, Karl-Schwarzschild-Str. 1, 85741 Garching, Germany \\ e-mail: martin@mpa-garching.mpg.de \\ 2 Institut für Theoretische Astrophysik, Universität Heidelberg, Albert-Überle-Str. 2, 69120 Heidelberg, Germany
}

Received 12 May 2005 / Accepted 23 August 2005

ABSTRACT

We describe an assembly of numerical tools to model the output data of the Planck satellite (Tauber 2004, Adv. Space Res., 34, 491). These start with the generation of a CMB sky in a chosen cosmology, add in various foreground sources, convolve the sky signal with arbitrary, even non-symmetric and polarised beam patterns, derive the time-ordered data streams measured by the detectors depending on the chosen satellitescanning strategy, and include noise signals for the individual detectors and electronic systems. The simulation products are needed to develop, verify, optimise, and characterise the accuracy and performance of all data processing and scientific analysis steps of the Planck mission, including data handling, data integrity checking, calibration, map making, physical component separation, and power spectrum estimation. In addition, the simulations allow detailed studies of the impact of many stochastic and systematic effects on the scientific results. Efficient implementation of the simulation allows extended statistics of signal variances and co-variances to be built up.

Although being developed specifically for the Planck mission, it is expected that the framework being employed, as well as most of the simulation tools, will be of use for other experiments and for CMB-related science in general.

Key words. cosmology: cosmic microwave background - cosmology: large-scale structure of Universe - methods: numerical

* Whole paper is only available in electronic form at http://www.edpsciences.org 


\section{Online Material}




\section{Introduction}

The simulation of realistic data streams is a very important task in the preparation for the Planck satellite mission. Scientifically, simulated data are required for a realistic assessment of the mission goals, and for planning how these goals can best be achieved. The performance of data-analysis algorithms and software needs to be tested against the scientific requirements of the mission. This pertains to methods for removing systematic noise from the maps, separating the multifrequency data into different physical emission processes, deriving both power spectra and higher-order statistical measures for CMB temperature and polarisation fluctuations, identifying "point" sources like distant galaxies and galaxy clusters, and much more. In order to develop, test, and calibrate methods for pursuing these scientific goals, full-sky maps need to be produced containing the $\mathrm{CMB}$ and all known components of foreground emission in the nine Planck frequency channels between 30 and $857 \mathrm{GHz}$, at the resolution of $5^{\prime}$ and the sensitivity of $\Delta T / T \sim 10^{-6}$ to be achieved by the Planck instruments. These maps need first to be "observed" following the scanning law foreseen for Planck and using realistic beam shapes, then filtered with the frequency and time response of Planck's detectors, and finally deteriorated by random and systematic instrumental noise before realistic scientific assessments can be made.

Also important is the use of simulated data for developing data-analysis techniques for the mission. At a resolution of $5^{\prime}$, and assuming sufficient oversampling of the beams, the full sky has approximately $5 \times 10^{7}$ pixels. Full-sky maps in all frequency bands and physical components (like foregrounds) need to be efficiently handled and stored. There will be 74 detectors on-board Planck, 52 on the High-Frequency Instrument (HFI), and 22 on the Low-Frequency Instrument (LFI). During one year of the mission, these detectors will produce something on the order of $10^{11}$ measurements along the slowly precessing, stepwise-circular scanning path from which the frequency maps need to be produced. Storing the data efficiently, preparing them for fast access, and handling them within the dataanalysis software pipelines needs to be thoroughly tested using data simulated at the correct temporal and spatial sampling rate.

Simulated data are also important for assessing mission operations and the technical planning. Depending on the scanning law, i.e. the path along which the satellite moves and scans the sky, the noise characteristics of the maps will be more or less anisotropic, because some regions on the sky will be observed much more often than others. At a fixed scanning law, the position of the detectors in the focal plane, their orientation relative to the optical axis, and the orientation of the optical axis relative to the spin axis of the satellite, all further affect the scientific goals by changing the noise characteristics, the coverage pattern on the sky, the quality of polarisation measurements, and more.

Finally, it is an important side-aspect of simulated data that they can be used to investigate the advantages and disadvantages of different models for data organisation and storage.
With single data sets exceeding the size of multiple GBytes, efficient data handling and processing requires that these data be stored in highly sophisticated ways, e.g. in data bases, such that sequential and random access to parts of the data is possible. As a European-based mission with many participating countries, Planck data analysis will operate in a distributed fashion (i.e. in several geographically separate data processing centres), which places further and stricter requirements on the way data are archived and retrieved.

The arguments above demonstrate that the simulation pipeline is essential for, and an integral part of, both the HFI and LFI Data Processing Centres (Pasian \& Sygnet 2002). Even before the Announcement of Opportunity for the Planck instruments was addressed, and actually during the phase-A studies for the mission, several simulations were made to understand the possible scientific outcome from Planck and to define the instrumental setup. The need was felt to put such a simulation effort on the same footing, and to extend it within a coordinated environment.

These considerations led to construction of the Planck simulation pipeline which began quite early in the project, approximately six years ago. At that time there were numerous simulation activities within many groups involved in the project, but those simulations were incomplete and were aiming at investigating a multitude of specific and different goals. They were widely used by different groups throughout the Planck consortia, but produced data in non-standardised and non-portable formats, and were not optimised with respect to their consumption of computer resources.

Starting with such existing simulation programmes, the Planck simulation pipeline was built according to the following design guidelines:

- The pipeline must be modular, i.e. different simulation tasks must be carried out by separate programmes, called modules. This simplifies extension of the pipeline by adding further simulation tasks as they become necessary, and it also facilitates testing and maintaining the pipeline and its modules.

- Data must be exchanged between modules in a simple, standardised, and machine-independent way. For the pipeline to be modular, the input-output interfaces of the modules are crucially important. For the interfaces to be simple and platform-independent, it was decided that data be exchanged in the FITS $^{1}$ format. It is foreseen that most of the data transfer between Planck simulation and dataanalysis modules will ultimately be done through a data base, but this does not change the principle of exchanging data through simple and platform-independent interfaces.

- Modules can be written in any suitable programming language. Since the only contact of a module to its environment is established through its interfaces, the internal implementation of the module is of no importance. Instead, allowing programmers to choose the language they find most suitable or convenient allows the pipeline to be rapidly extended.

\footnotetext{
${ }^{1}$ http://archive.stsci.edu/fits/fits_standard/
} 
Following these criteria, construction of the Planck simulation pipeline proceeded in four steps:

- First, simulation programmes were collected within the Planck consortia, converted to modules in the sense that they were enclosed within suitable interfaces, and augmented by missing modules specifically written to complete the pipeline. In that way, it was possible within three months to produce the first simulated time-ordered data streams from full-sky temperature maps of the CMB and several foreground components, which were convolved with realistic beams along the scanning path, filtered according to the frequency response, then appropriately timesampled, and had random noise added.

- Second, the function of the pipeline was extended by adding standard-compliant modules necessary to simulate observation of polarised signal components, point sources, systematic noise, extended beam wings, and the CMB dipole, taking the exact motion of the spacecraft into account. Massive production of simulated data was started; e.g. time-ordered data were produced for different detectors, scanning strategies and combinations of foregrounds.

- In a third phase, the Planck pipeline was consolidated by improving all individual modules in terms of their algorithms and their implementation and by adding modules that simulate higher-order effects like the emission of moving point sources or the detailed motion and rotation of the spacecraft. In parallel, a large part of the code base of the pipeline was ported from several programming languages to $\mathrm{C}++$.

- Finally, the entire code was optimised in the sense that memory consumption was drastically reduced, computation speed was strongly increased, OpenMP parallelisation $^{2}$ was added where possible, common functions used by more than one module were bundled in central libraries, and strict adherence to language standards was enforced for compiler-independence.

As a result, there is now a simulation pipeline capable of producing realistic data streams for a full year of observation with individual Planck detectors within a day of CPU time on a computer with sufficiently large main memory $(\approx 10 \mathrm{~GB})$. For near-realistic parameter choices, the entire pipeline can be run successfully on standard PCs. Due to the strict adherence to language standards and avoidance of platform-specific libraries, the package cleanly compiles on four different types of UNIX platforms in both 32- and 64-bit architectures. Furthermore, it has proven suitable to run on a computational grid infrastructure (Taffoni et al. 2005). The pipeline code is freely available within the Planck consortium.

After giving a brief overview of the Planck mission in Sect. 2 , in Sect. 3 we describe the layout of the pipeline, i.e. the decomposition of its simulation tasks into modules, and then in Sect. 4 the data it produces. Sections 5 and 6 list the pipeline components common to all modules and the individual simulation modules themselves. A summary is presented in Sect. 7.

\footnotetext{
${ }^{2}$ http://openmp.org
}

\section{Summary of Planck's characteristics}

Here we briefly summarise some characteristics of the Planck mission that are crucial to the design and construction of the simulation pipeline and to understanding its function.

Planck will observe the microwave sky from a Lissajou orbit centred on the outer (and unstable) Lagrangian point L2 of the Sun-Earth/Moon system, $1.5 \times 10^{6} \mathrm{~km}$ from Earth. This enables the spacecraft to always have the Earth and the Sun at its back, and have the Moon at a small angle relative to its backward direction. Planck will be spin-stabilised, rotating at 1 r.p.m. The optical axis of its Gregorian telescope points at an almost right angle with the rotation axis, thus scanning the sky along circles. The rotation axis will be kept fixed for $60 \mathrm{~min}$ and then repointed by 2.5 arcmin such that Planck progresses as the Earth moves around the Sun. Planck will thus cover the full sky in half a year. The full mission (after reaching L2) is expected to last 18 months.

The rotation is governed by the inertial tensor of the spacecraft, which is time-dependent due to fuel and coolant consumption. Repointing the spacecraft every hour causes precession, which needs to be damped away at the beginning of each pointing period.

The angle between the optical and rotation axes is slighly less than $90^{\circ}$. For Planck to cover the full sky, its rotation axis will make slow excursions from the Ecliptic plane with an amplitude of several degrees. This scanning pattern implies that the sky will be covered inhomogeneously, less dense near the Ecliptic and more dense along lozenge-shaped curves centred on the Ecliptic poles. Rings on the sky scanned in consecutive pointing periods will intersect, allowing cross calibration. The scanning pattern also implies that contact with the receiving antenna in Perth (Australia) will be limited to two hours per day, during which data down- and uploads will have to be completed.

Two instruments will receive the incoming microwave radiation. The low-frequency instrument (LFI) has high electron mobility transistors (HEMTs) as detectors, allowing measurement of amplitudes and phases of incoming waves, and thus of their intensity and polarisation. It has three channels at 30, 44, and $70 \mathrm{GHz}$. The high-frequency instrument (HFI) uses bolometers instead. Four of its channels at 100, 143, 217 , and $353 \mathrm{GHz}$ are polarisation-sensitive, while the highestfrequency ones at 545 and $857 \mathrm{GHz}$ are not. Passive cooling is insufficient for these instruments. LFI will operate at $20 \mathrm{~K}$, while HFI needs to be cooled down to $100 \mathrm{mK}$. This will be achieved by a four-stage cooling system. Residual thermal coupling between the cooling system, in particular the sorption cooler, and the detector electronics, the instruments, and the telescope gives rise to a noise component that varies with the cyclic operation of the cooler.

The angular resolution of the beams decreases from about 30 to 5 arcmin from the 30 to the $857 \mathrm{GHz}$ channels. While the cores of the beams have approximate Gaussian profiles with slightly elliptical cross sections, their wings are extended and can receive weak signals from regions of the sky that are quite far away from the direction of the beam core. These socalled far-side beam lobes thus add a weak noise contribution. 
The feed horns of the individual detectors are slightly inclined from their positions in the focal plane towards the optical axis of the telescope, which implies that at a given time all detectors are observing different points on the sky.

The channel width is approximately $20 \%$ of the central frequency for LFI and $30 \%$ for HFI. During scans, the signal received by the detectors will be integrated over certain time intervals and read out at frequencies varying between 30 and $200 \mathrm{~Hz}$. The sampling frequency needs to be high enough for the beam to proceed sufficiently less than its own width along the scanning path during one sampling period.

Slow gain drifts in the detector sensitivity cause Planck's sensitivity to vary slowly along the circular scan paths. The frequency power spectrum of these gain drifts falls approximately as a power law in frequency and is thus called $1 / f$ noise. This noise component gives rise to stripe patterns in the measurements that follow the scan circles on the sky.

\section{Pipeline layout}

Figure 1 illustrates a typical pipeline setup used for simulating detector timelines and pointings starting from basic inputs like cosmological parameters, microwave emission models for galactic and non-galactic components, and a scanning strategy. A short description of the individual steps is given below:

- Initially, a CMB power spectrum is generated from a set of assumed cosmological parameters, using the CAMB code by Lewis and Challinor (see Sect. 6.1; Lewis et al. 2000).

- A particular realisation of this power spectrum is generated using the syn_alm_cxx module (Sect. 6.2) in terms of a set of spherical-harmonic coefficients $\left(a_{l m}\right)$.

- The available set of diffuse foreground component maps is transformed into spherical-harmonic coefficients using the anafast_cxx module (Sect. 6.2).

- A combined set of $a_{l m}$ coefficients is generated using the almmixer module, weighting the individual component maps by convolving their electromagnetic spectra with the frequency response of the chosen Planck detector (Sect. 6.3).

- A spherical-harmonic transform of an idealised beam template is generated using the gaussbeampol module; alternatively, a realistic, simulated beam pattern can be expanded into spherical harmonics using beam $2 \mathrm{alm}$ (Sect. 6.4).

- Since a real-space representation of the beam is required for efficient convolution with point-sources, the alm2grid code (Sect. 6.2) is used to generate a beam profile sampled on a grid which is equidistant in the polar angles $(\vartheta, \varphi)$.

- Using the spherical-harmonic coefficients of the combined sky and the beam, the totalconvolve_cxx module produces a so-called ring set (see Sect. 4), from which the intensity measured by the detector can be determined for all detector pointings and orientations (Sect. 6.5).

- The simmission module produces a time stream of satellite locations and orientations from input values describing the mission orbit parameters, the scanning strategy, and the dynamical properties of the satellite (Sect. 6.6).

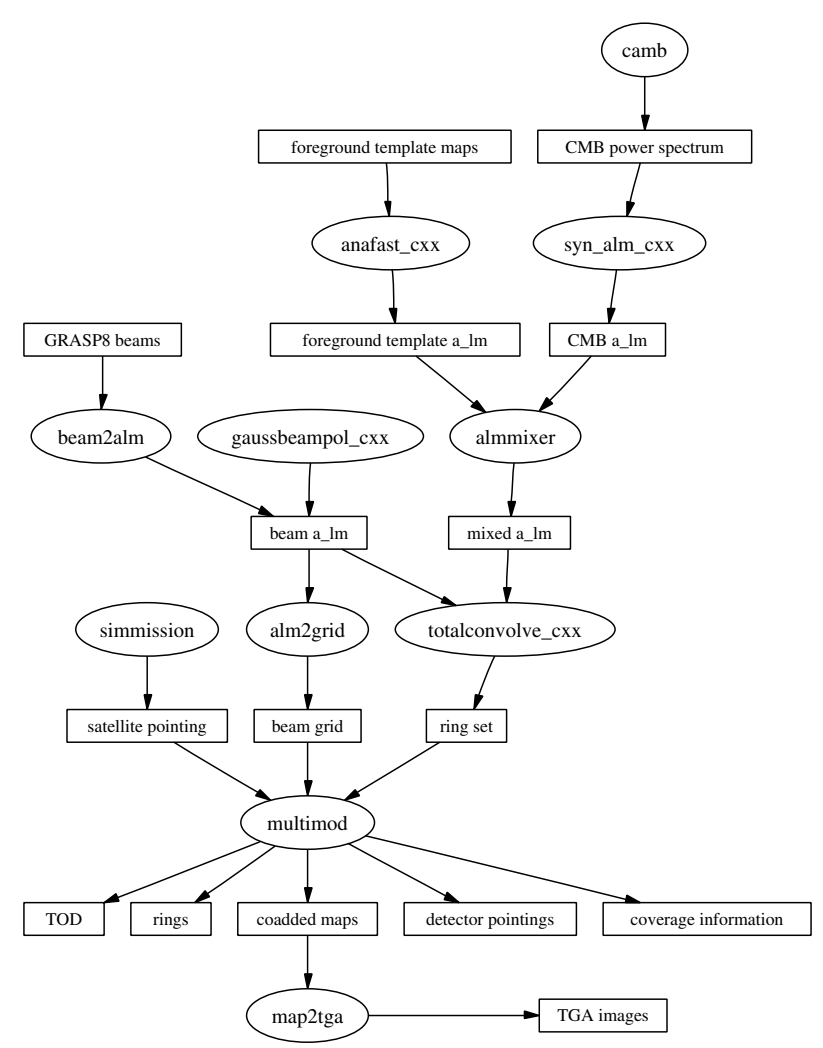

Fig. 1. Schematic data flow in a typical Planck simulation pipeline. Rectangular components denote parameters or data products (see Sect. 4), whereas elliptic shapes represent modules (see Sect. 6).

Additionally, simmission can generate data tables containing the location of the planets as seen from the spacecraft, which are used by the point-source convolver.

- The multimod code (Sect. 6.7) processes the ring sets, satellite pointing information, and beam profiles, and it generates time streams containing detector pointings and the measured signal. In addition to scanning the convolved diffuse sky signal (Sect. 6.7.2), multimod also computes the signal of the CMB dipole (Sect. 6.7.3), the pointsource signal (Sect. 6.7.4), the noise generated by the sorption cooler on-board the spacecraft (Sect. 6.7.5), and the instrumental $1 / f$-noise (Sect. 6.7.7). The sampling and integration techniques used for the individual detectors (Sect. 6.7.6) are also taken into account.

Mainly for diagnostic purposes, multimod can also generate co-added sky maps (produced by simply adding every simulated sample to the pixel containing the corresponding pointing and subsequent averaging) and coverage maps in HEALPix $^{3}$ format, which can in turn be converted to Targa ${ }^{4}$ image files by the map2tga programme.

It is evident that, in this layout, the pipeline becomes increasingly mission-specific from beginning to end. While the calculation of the CMB power spectrum and the creation of a set of $a_{l m}$ is completely independent of the mission setup,

\footnotetext{
http://healpix.jpl.nasa.gov

4 http://astronomy.swin.edu.au/ pbourke/dataformats/ tga/
} 
the almmixer module already needs information about the detectors' frequency response. For the total convolution step, the geometrical beam properties must be known as well, and in order to produce time-ordered data, a detailed scanning strategy and quantities like the satellite's inertial tensor must be specified to the simmission module. The multimod programme also requires the detectors' noise characteristics, sampling frequencies, and integration time constants.

Conceptually, the simulation process can be subdivided into two parts. All modules up to and including the totalconvolver and beam-generation modules produce whole-sky data without time dependence; the subsequent modules generate time-dependent, localised output.

It is important to note that the layout described here is by no means fixed, but can be adjusted to whatever is needed to perform a particular simulation task. This can be achieved by using the pipeline editor code, which is part of the Planck process coordinator package.

The following sections give a more in-depth description of the data types and codes constituting the simulation pipeline. While this documentation should suffice to decide in which situations a given module or data type can be used, it does not give detailed instructions, such as lists of mandatory parameters. This topic is covered by the document "Compilation and Usage of the Planck Simulation Modules", available in Planck's LiveLink repository or from the authors, which serves as a detailed manual for practical use of the simulation package.

\section{Data products}

This section documents the various types of data sets produced by the Planck simulation package, which are intended for further use by the Planck Data Processing Centres.

Unless otherwise stated, all data products of the Planck simulation package containing intensities are stored as antenna temperatures measured in Kelvin. Angles are generally stored as radians. The simulation pipeline produces maps and pointing information in ecliptic coordinates.

Maps:

The Planck simulation package uses maps in the HEALPix pixelisation scheme to store full-sky information like CMB intensity and polarisation, foreground components, sky coverage information, and co-added maps. In addition to single-component maps, three-component maps are used to store polarised data in the form of the Stokes $I, Q$, and $U$ parameters.

Spherical-harmonic coefficients $\left(a_{l m}\right)$ :

A band-limited function $f(\vartheta, \varphi)$ with maximum multipole order $l_{\max }$ defined on the sphere can be expressed as a set of spherical-harmonic coefficients $a_{l m}$, which is implicitly defined by

$f(\vartheta, \varphi)=\sum_{l=0}^{l_{\max }} \sum_{m=-l}^{l} a_{l m} Y_{l m}(\vartheta, \varphi)$.

These complex coefficients are stored in a three-column format, where the first column holds the integer index $l^{2}+l+m+1$, which unambiguously encodes both $l$ and $m$ because $|m| \leq l$. The second and third columns contain the real and imaginary parts, respectively, of the corresponding coefficient. Since the Planck simulation package exclusively works on real-valued maps, coefficients with negative $m$ are not stored because of the condition $a_{l,-m}=a_{l m}^{*}$ for real-valued functions on the sphere. The index column is assumed to contain monotonically increasing values, and coefficients not listed in the table are assumed to be zero. For polarisation information, which can also be stored in the form of spherical-harmonic coefficients, the relations between real-space and spherical-harmonic space are more involved because the $Q$ and $U$ Stokes parameters are quantities of spin \pm 2 , and must therefore be expanded in spin-weighted harmonics ${ }_{ \pm 2} Y_{l m}$. Their exact definition can be found in Zaldarriaga \& Seljak (1997).

Power spectra $\left(C_{l}\right)$ :

The power spectra read and generated by the Planck simulation modules consist of either one or four components: both variants contain the auto-correlation $C_{l, T T}$ of the temperature fluctuations. The four-component variant also contains the auto-correlations $C_{l, \mathrm{EE}}$ and $C_{l, \mathrm{BB}}$ of the $\mathrm{E}$ and B polarisation modes, as well as the cross-correlation $C_{l, \mathrm{TE}}$ between temperature and E-type polarisation. The following normalisation conventions are used:

$C_{l, a a}=\frac{1}{2 l+1} \sum_{m=-l}^{l}\left|a_{l m}\right|^{2} \quad$ (autocorrelation)

and

$C_{l, a b}=\frac{1}{2 l+1} \sum_{m=-l}^{l} a_{l m}^{*} b_{l m} \quad$ (crosscorrelation).

Time-ordered data:

The time-dependent output signal of the detectors is stored as a single column of data. Since data streams for the complete mission can become very large, and many computer platforms do not support files larger than 2 GBytes, the Planck simulation modules can optionally split time-ordered data into several files.

Detector pointings:

This time-ordered quantity is stored in a two- or threecolumn format. The first two columns describe the co-latitude and the longitude of the beam centre (in radians), while the optional third column contains the beam orientation, given as the angle between the tangent to the local meridian (pointing northwards) and the $x$-axis of the rotated beam coordinate system. Similar to the time-ordered data, detector pointings can be split into several files to avoid the 2-GByte limit of the file size.

Detector information:

Several Planck simulation modules need information about Planck's detectors such as their position in the focal plane, the orientation of their optical axes relative to the telescope's optical axis, their noise characteristics, 
spectral responses, sampling properties, ellipticities, etc. This information is centrally stored and maintained in the so-called focal plane database, which was originally a text file, but has now been converted to a FITS table for use within the Planck simulation package. Recently, a new tool was added which allows extraction of the required detector information from HFI's Instrument Model Object (IMO) files and its conversion to FITS. In addition to the detector characteristics, the focal-plane database also contains the angle between the nominal spin axis of the satellite and the optical axis of the telescope.

\section{Ring sets:}

This data type is written by the totalconvolver module (Sect. 6.5) and used by the interpolator module (Sect. 6.7.2). It contains the three-dimensional array

$$
T\left(\phi_{\mathrm{E}}, \phi, m^{\prime \prime}\right)=\sum_{m, m^{\prime}=-l_{\max }}^{l_{\max }} T_{m, m^{\prime}, m^{\prime \prime}} \mathrm{e}^{i m \phi_{E}+i m^{\prime} \phi},
$$

where $\phi_{\mathrm{E}}, \phi$ and $T_{m, m^{\prime}, m^{\prime \prime}}$ are defined in Eq. (8) of Wandelt \& Górski (2001). Our implementation of the algorithm does not carry out the Fourier transform over the $m^{\prime \prime}$ direction because the necessary interpolation can be carried out more easily in this representation (see Sect. 6.7.2 for details).

For real-valued skies and beams, the condition $T\left(\phi_{\mathrm{E}}, \phi, m^{\prime \prime}\right)=T^{*}\left(\phi_{\mathrm{E}}, \phi,-m^{\prime \prime}\right)$ is fulfilled, so the coefficients with negative $m^{\prime \prime}$ and the imaginary part of $T\left(\phi_{\mathrm{E}}, \phi, 0\right)$ need not be stored.

\section{Common components}

\subsection{External libraries}

\subsubsection{FITS I/O}

Since all modules are presently communicating via FITS files (NOST 1999), the cfitsio library (Pence 1999) is required for the pipeline to operate. Currently, version 2.510 of the library is used. In the future, it is planned to store the data in data-base system.

\subsubsection{FFT}

Several modules need to perform fast Fourier transformations, which can be divided into two different usage scenarios:

- Most codes (like the transformations of maps between real and spherical-harmonic space carried out within the HEALPix package) perform FFTs of many different lengths, but each FFT of a given length is only performed a few times. Here the Planck simulation package contains a port to the $C$ language of the FFTPACK code originally developed by Swarztrauber (1982). If the length of the required FFT has very large prime factors, Bluestein's algorithm (Bluestein 1968) is employed; the choice of the algorithm occurs automatically. Both complex- and real-valued transforms are supported.
- Several other, still experimental, modules require a highly optimised FFT transform of a given length, which is executed many times. This task is handled by the FFTW library (Frigo \& Johnson 1998), of which the Planck simulation package currently uses version 3.0.1.

\subsection{Other common functions}

Apart from using externally developed general-purpose software such as libcfitsio, the codes have many additional commonalities: they perform file input and output in a very similar way (using only a small subset of the cfitsio function), they deal with a common set of data types (see Sect. 4), and they need to operate on them in similar ways. Keeping a separate implementation of these facilities for each module would cause unnecessary code duplication, leading to software that is both error-prone and hard to maintain. For this reason, common functions are implemented exactly once and linked to all programmes that need it. However, since some modules in the Planck simulation package are written in Fortran 90 and others in $\mathrm{C}++$, a few common components (most notably the support functions concerned with data $\mathrm{I} / \mathrm{O}$ ) had to be implemented in both languages to allow convenient usage.

\subsubsection{Random number generator}

Several modules, most notably the $1 / f$-noise generator and the syn_alm_cxx module, which creates sets of sphericalharmonic coefficients from a power spectrum, require streams of random numbers. Using the default random-number generators supplied with the programming language is not appropriate in our situation, since the period length and overall quality of these random numbers depend on the operating system and the compiler, whereas the data products created with the Planck simulation package should be identical on all supported platforms (for identical random seeds).

Currently, the Planck simulation modules use a generator of the xorshift type (Marsaglia 2003) with a period of $2^{128}-1$, which is more than sufficient for the necessary calculations. In addition to its excellent randomness properties, the algorithm is also very efficient. And since most codes require random numbers with a Gaussian distribution instead of uniform ones, the simulation package contains an implementation of the BoxMuller method (Box \& Muller 1958) for generating the former from the latter.

\subsubsection{Input of simulation parameters}

All Planck simulation modules follow a single convention for obtaining simulation parameters (like cosmological parameters, map resolution, detector names, names of input and output files, etc.) from the environment. This is done via plain ASCII files obeying a simple format. Each parameter is defined in a separate line using the form

[name $=$ [value $]$

Empty lines are skipped. Lines starting with a hash (\#) sign are ignored and can thus be used as comment lines. 


\subsubsection{Handling of data $\mathrm{I} / \mathrm{O}$}

Data exchange between the various pipeline modules is performed exclusively via FITS files. Although the cfitsio library provides a comprehensive interface for creating and reading these files, calling it directly from the modules is in most cases more complicated and error-prone than necessary for the limited set of data types used within the Planck simulation package. To simplify data input and output for the module author, a powerful but fairly easy-to-use set of "wrapper" functions around cfitsio was written in both Fortran 90 and $\mathrm{C}++$, which supports all input and output operations performed by the Planck simulation modules.

This abstraction from the interface of the underlying library has the additional advantage that the external data format can be changed with relatively little impact on the module code, simply by adjusting the implementation (without modifying the user-visible interface) of the common input-output functions mentioned above to the new format. The anticipated switch from FITS files to the Planck Data Management Component as a means for transporting data between modules will greatly benefit from this design.

On an even higher level, functions exist which read and write entire maps, spherical-harmonic coefficient sets and power spectrum objects; this code is also used by many modules.

\section{Module descriptions}

In the following subsections, we describe the function of all modules in the Planck simulation package. Where possible, we only give a short description of the underlying algorithms and cite the original works introducing the numerical methods; if no such publication exists, we provide a more detailed description. For the most resource-consuming applications, we also specify their CPU, memory, and disk-space requirements, as well as their scaling behaviour with regard to relevant input parameters (such as the map resolution or the maximum multipole order $\left.l_{\max }\right)$. Parallelisation of a code is also mentioned where applicable.

\section{1. $C A M B$}

For generating CMB power spectra, the November 2004 version of the $\mathrm{CAMB}^{5}$ code (Lewis et al. 2000) is used. This code is based on CMBfast (Seljak \& Zaldarriaga 1996), but is more portable and can be integrated more easily into the Planck simulation package, mainly because it does not rely on unformatted files for storage of intermediate data products.

Memory and CPU requirements of the CAMB code are rather modest for the parameter sets typically used for Planck simulations (memory consumption $<100 \mathrm{MB}$, runtime $<1 \mathrm{~min}$ ), hence it can be run without any problems on desktop machines.

\footnotetext{
5 http://camb.info
}

\subsection{HEALPIX}

The Planck simulation pipeline makes extensive use of the HEALPix pixelisation of the sphere (Górski et al. 2002, 2005). It is implemented in a software package which contains many associated algorithms, among them some exploiting the efficiency of the HEALPix scheme for spherical-harmonic transforms. The main usage areas of the package in the simulation pipeline are:

- generating spherical-harmonic coefficients from power spectra $C_{l}$;

- conversion between $a_{l m}$ and full-sky maps;

- generation of co-added maps from time-ordered data;

- generation of efficient look-up tables for the point-source convolver.

A large part of the HEALPix software package, which is available in Fortran 90, was re-implemented in $\mathrm{C}++$ in order to be conveniently accessible from within the $\mathrm{C}++$ simulation modules. During this migration, the function of the synfast tool, which essentially generates sky maps from power spectra, was split into two separate facilities, syn_alm_cxx (which generates $a_{l m}$ from a power spectrum) and alm2map_cxx (which transforms $a_{l m}$ coefficients to a HEALPix map). As can be seen in Fig. 1, this allows feeding the results of syn_alm_cxx directly into the almmixer module (Sect. 6.3), and avoids the conversion to real space and back to $a_{l m}$, which was necessary in earlier versions of the Planck simulation package and caused some avoidable loss of accuracy and waste of computer resources.

The ported code (especially the conversions between maps and $a_{l m}$ coefficients) was optimised with regard to memory and CPU time consumption. On shared-memory computers, OpenMP parallelisation is used.

The alm2grid programme is an addition to the existing HEALPix facilities. This code converts $a_{l m}$ coefficients to values on an equidistant grid in the polar angles $(\vartheta, \varphi)$, whose extent in $\vartheta$ can be chosen freely. Using this format, it is easy to calculate a high-resolution real-space representation of a beam pattern, which in turn is a necessary input for the point-source convolver. The $\mathrm{C}++$ package also contains a testsuite to validate the correctness and accuracy of the ported code.

Starting with version 2.0, the official HEALPix package contains the described $\mathrm{C}++$ modules, and is distributed under the terms of the GNU General Public License ${ }^{6}$.

\subsection{The skymixer/almmixer}

The skymixer component serves two purposes:

- It converts maps of the CMB and diffuse foreground sources (such as the Galactic synchrotron and dust emission and the thermal and kinetic Sunyaev-Zel'dovich effects) from the units in which they are typically provided (e.g. $\mathrm{MJy} / \mathrm{sr}$ at a certain frequency for the Galactic maps, and the Compton $y$-parameter for the thermal Sunyaev-Zel'dovich maps) to antenna temperature observed by a given Planck

\footnotetext{
${ }^{6}$ http://www.gnu.org
} 
detector. During this conversion, the frequency response of Planck's detectors is taken into account, i.e. the frequencydependent sky signal is convolved with the sensitivity of the detectors not only at the nominal detector frequency, but also in a frequency band around it. Currently, the spectral detector response can have Gaussian, top hat or $\delta$ form.

- It adds the resulting antenna-temperature maps to a single map with all the selected components.

The currently employed code is a re-implementation of a version by R. Ansari, which was based on the SOPHYA ${ }^{7}$ library.

The microwave sources currently supported by skymixer are:

- CMB given as temperature fluctuations in Kelvin:

This kind of map is typically generated from a power spectrum $C_{l}$ produced by the CAMB code.

- Galactic synchrotron emission, given in $\mathrm{MJy} / \mathrm{sr}$ at $408 \mathrm{MHz}$ :

The template was provided by G. Giardino; details concerning its creation are given by Giardino et al. (2002). This component is extrapolated to the Planck frequency bands by using a power-law spectrum with an exponent of -0.75 between $408 \mathrm{MHz}$ and $22 \mathrm{GHz}$, and with an exponent of -1.25 above $22 \mathrm{GHz}$.

A spectral-index map derived from Wilkinson-MAP data is also available, but has not yet been included in the extrapolation algorithm.

- Galactic dust emission given in MJy/sr at $100 \mu \mathrm{m}$ :

Currently this source is handled by a one-component approximation provided by $\mathrm{C}$. Baccigalupi. The full twocomponent model presented by Finkbeiner et al. (1999) is currently being implemented.

The employed template map was published by Schlegel et al. (1998).

- Galactic free-free emission:

This map was derived from a template map of galactic $\mathrm{H}_{\alpha}$-emission published by Finkbeiner (2003), according to the model of Valls-Gabaud (1998).

- thermal and kinetic SZ-effect, given as the Compton- $y$ and Compton- $w$ parameter, respectively:

Briefly, these maps were produced using the galaxy-cluster distribution in space, peculiar velocity and redshift found in the Hubble Volume simulation (Jenkins et al. 2001; Colberg et al. 2000), attaching appropriately scaled gas-dynamical cluster simulations to the cluster positions and projecting the Compton- $y$ and $w$ parameters on the sphere. Details about the template maps are given by Schäfer et al. (2004a). Additional maps for the thermal and kinetic SZ-effect of the local super cluster structure have become available recently. In this case, the full sky SZ maps are directly calculated from a hydodynamical, constrained simulation of the local universe (Dolag et al. 2005), which spans a sphere of $110 \mathrm{Mpc}$ radius. These maps are complemented by considering the galaxy-cluster distribution outside this sphere using the Hubble Volume simulation as described before.
- Emission by rotating CO molecules:

This foreground component only contributes to the HFI channels. The template map was published by Dame et al. (1996, 2001). We use the method given by Schäfer et al. (2004b) to determine the intensities in Planck's frequency bands.

Since the output of the skymixer is most frequently used in a subsequent run of the totalconvolve_cxx module (see Sect. 6.5) which requires $a_{l m}$ coefficients as input, and since the synthesised CMB signal can be very efficiently created in the form of spherical-harmonic coefficients $a_{l m}$, an additional variant of the skymixer code was implemented. This code (named almmixer) performs exactly the same task as skymixer, but operates entirely in spherical-harmonic space. Using this code eliminates the need for converting between angular and spherical-harmonic space twice during a run of a typical simulation pipeline and thus saves a considerable amount of CPU time.

Both codes allow the suppression of the CMB monopole in the output, which is usually not needed by any subsequent analysis and which would decrease the numerical accuracy of the output.

The runtime of both programmes is rather short and dominated by the input and output operations. The memory consumption of the skymixer is low (below 10 MBytes) and independent of the map size, since it processes them chunk by chunk. In contrast, the almmixer module holds two $a_{l m}$ sets in memory, so that it requires approximately $16 l_{\max }^{2}$ bytes of storage.

\subsection{Beam generation}

Several Planck simulation modules require information about the beam shapes of Planck's detectors, typically in the form of a set of spherical-harmonic coefficients.

One way to obtain these is the module gaussbeampol, which takes a detector name as input, looks up the detector's beam characteristics in the focal-plane database, and produces the corresponding $a_{l m}$ coefficients. The gaussbeampol module can generate axisymmetric or elliptical Gaussian beams with and without polarisation, and allows arbitrary orientations for polarisation and ellipticity.

Realistic beam patterns are often delivered in the GRASP8 format $^{8}$, which in turn consists of the GRD and CUT subformats. The beam $2 \mathrm{alm}$ code can convert beam files of both sub-formats to $a_{l m}$ coefficients, and also allows combining two GRASP8 files (containing the full and main beam, respectively) to a single $a_{l m}$ set.

The point-source convolver requires a real-space representation of the beam, which can be obtained from the $a_{l m}$ coefficients using the alm2grid or alm2map_cxx modules.

\footnotetext{
${ }^{8}$ http://www.ticra.com
} 


\subsection{Total convolution}

This module takes as input the spherical-harmonic coefficients of a sky map and a beam, and computes the convolution of sky and beam for all possible directions $(\vartheta, \varphi)$ and orientations $(\psi)$ of the beam relative to the sky. Both unpolarised and polarised convolutions are supported. In the polarised case, three sets of spherical harmonics are required for sky and beam, respectively, for their Stokes- $I, Q$, and $U$ parameters. The output then consists of the sum of the three separate convolutions.

Our implementation is analogous to the algorithm presented by Wandelt \& Górski (2001), including the extensions for polarisation given by Challinor et al. (2000). However, the code was modified to allow complete shared-memory parallelisation of all CPU-intensive tasks (starting from the partial parallelisation contributed by Stephane Colombi), and the symmetries inherent in the matrix $d_{m m^{\prime}}^{l}$ (introduced in Eq. (6) of Wandelt \& Górski 2001) were used for further CPU and memory savings. With the new code, convolutions up to $l_{\max }=1500$ and $m_{\max }=2$ can be done in less than five minutes on a modern desktop computer.

The resource consumption of the totalconvolve_cxx module scales roughly with $l_{\max }^{2} m_{\max }$ for memory and $l_{\max }^{3} m_{\max }$ for CPU time. Although the code is fully parallelised, it will most likely not scale very well, since the cache re-use of the algorithm is poor and a saturation of the memory bandwidth will limit overall performance.

The output consists of the three-dimensional complexvalued array $T(\vartheta, \varphi, m)$, which contains $l_{\max }+1+x$ points in $\vartheta$-direction ( $x$ is a small number of additional points needed for interpolation purposes), $2 l_{\max }+1$ points in $\varphi$-direction, and $m_{\max }+1$ values in $m$-direction. In contrast to the original implementation by B. Wandelt, the final FFT is only carried out over $\vartheta$ - and $\varphi$-directions, because interpolation is easier for this case; see also Sect. 6.7.2. To increase the spatial resolution of the output ring set, the working array can be zero-padded to any desired $l_{\max \text {,out }} \geq l_{\max }$ before the FFT is performed.

\subsection{Satellite dynamics}

The Planck simulation package makes use of the simmission code developed by Floor van Leeuwen and Daniel Mortlock (van Leeuwen et al. 2002; Challinor et al. 2002), which takes a wide variety of mission-related parameters as input like the exact orbit around the outer Lagrangian point L2 of the SunEarth/Moon system, the scanning strategy, the satellite's inertial tensor, etc.

The most important output of the simmission module is a table containing the position and orientation of the satellite in fixed time intervals during the entire mission. In addition, it optionally calculates the positions of the Sun and planets relative to the Planck spacecraft, which is important for the pointsource convolver.

\section{7. multimod}

The multimod code comprises most of the functions offered by the Planck simulation package for creating and manipulating time-ordered information. This includes creation of detector pointings and signal time streams from various sources like the CMB dipole, point sources, and detector noise.

At first glance, this approach appears to contradict the goal of modularity stated at the beginning of this paper, which would suggest writing many small pieces of code implementing a single task each. This strategy, however, would have a negative impact on performance, since in most of the simulation codes working on time-ordered data, the disk input or output times are at least comparable to the computations themselves. Combining all the effects in one programme allows us to read and write the data only once and perform all manipulations without storing intermediate data products on disk.

Even though all the functions are linked together into one executable, the various components are still implemented in a very modular fashion, so that they can be easily used to build smaller modules with reduced generality. For example, the Planck simulation package contains a stand-alone pointsource convolution code, which includes exactly the same $\mathrm{C}++$ classes also used by the multimod code.

The multimod programme processes data in chunks of one pointing period at a time, which corresponds to an hour of measurement. This keeps the amount of main memory occupied by time-ordered data rather low. On the other hand, a pointing period still contains enough individual samples to allow efficient OpenMP-parallelisation of the code.

Schematically, multimod performs the following steps for each pointing period:

1. Calculate the detector pointings at a sampling rate of ringres/60s from the satellite pointings (see Sect. 6.7.1).

2. Calculate and add all of the requested signal contributions (except for $1 / f$-noise) at a sampling rate of ringres $/ 60 \mathrm{~s}$ (see Sects. 6.7.2-6.7.5).

3. Process the calculated signal in the sampler module (see Sect. 6.7.6).

4. Add the $1 / f$-noise if requested (see Sect. 6.7.7).

5. Produce the detector pointings at the detector sampling rate.

6. Write the requested signal and pointing streams to disk.

7. Update the coverage and coadded sky maps.

Each of these steps is only executed if its output was requested by the user or if it is required by subsequent steps; i.e. if the user only asks for the generation of detector pointings, only steps 5 and (partially) 6 are executed.

The parameter ringres can be chosen freely by the user; it determines the sampling rate used for producing the "ideal" detector signal (i.e. the signal before the modification by the detector electronics and the sampling process). In theory this 
sampling rate should be infinitely high; for practical purposes it should be sufficient to choose ringres/60 $\mathrm{s} \approx f_{\text {samp }}$ for smooth signals (like the CMB and galactic foregrounds) and ringres $/ 60 \mathrm{~s} \approx 3 f_{\text {samp }}$ for the $\mathrm{SZ}$ and point-source signals.

\subsubsection{Detector pointings}

Using the time stream $M_{n}$ of satellite orientations generated by the simmission module, the detector-pointing component produces time-ordered detector pointings at arbitrary sampling rates. For this purpose, it is necessary to determine the satellite orientation at any given point in time. This is achieved by extracting the rotation matrix $R_{n}$ between every $M_{n}$ and $M_{n+1}$. From this matrix, an axis $\boldsymbol{r}_{n}$ and angle $\alpha_{n}$ of rotation can be computed. The satellite orientation at a time $t$ with $t_{n} \leq t<t_{n+1}$ can then be approximated by

$M_{t}=R M\left(r_{n}, \frac{t-t_{n}}{t_{n+1}-t_{n}} \alpha_{n}\right) M_{n}$

where $R M(\boldsymbol{r}, \alpha)$ is a rotation matrix performing a rotation of $\alpha$ around the axis $\boldsymbol{r}$.

After this rotation interpolation, another rotation matrix must be applied, which describes the detector position and orientation relative to the satellite coordinate frame. This matrix depends on the angle between the optical axis and the nominal spin axis, as well as on the detector-specific angles $\phi_{u v}$, $\vartheta_{u v}$, and $\psi_{u v}$ given in the focal-plane database, which specify the position and orientation of all detector horns relative to the coordinate frame of the focal plane.

\subsubsection{Interpolator}

The interpolator module's task is to determine the radiation intensity falling onto a detector for a given co-latitude $\vartheta$, longitude $\varphi$, and beam orientation $\psi$. To accomplish this, the output of the totalconvolve_cxx module for the desired sky signal and the detector's beam is used. As Wandelt \& Górski (2001) point out, this dataset is sufficient (for a given $l_{\max }$ and $m_{\max }$ of the beam) to reconstruct the exact signal for any $(\vartheta, \varphi$, $\psi)$-triple. However, exact evaluation is prohibitively expensive in practice, since it would involve a sum over $l_{\max }^{2} m_{\max }$ terms for a single data point.

The approach used in the interpolator is based on the assumption that $l_{\max }$ will be rather large $(\approx 4000)$ for realistic simulations, while the beam's $m_{\max }$ is usually not larger than 20 ; i.e. the beam pattern has no high-frequency azimuthal asymmetries. The high $l_{\max }$ implies a fairly high resolution in $\vartheta$ and $\varphi$ for the totalconvolver's output, so that polynomial interpolation, whose order can be chosen via a parameter, can be used for those two directions. In the $\psi$-direction, this approach is not accurate enough because of the sparse sampling, so that the Fourier sum of the $\psi$-components must be calculated:

$S(\vartheta, \varphi, \psi)=\sum_{m=-m_{\max }}^{m_{\max }} T_{\text {interpol }}(\vartheta, \varphi, m) \mathrm{e}^{\mathrm{i} m \psi}$.

A straightforward calculation of $\mathrm{e}^{\mathrm{i} m \psi}$ would be quite CPUintensive because of the required trigonometric function calls, but making use of the trivial recursion relation $\mathrm{e}^{\mathrm{i}(m+1) \psi}=$ $\mathrm{e}^{\mathrm{i} m \psi} \mathrm{e}^{\mathrm{i} \psi}$ reduces calculation time significantly. This optimisation is only applicable here because $m_{\max }$ cannot become very large; if it did, the roundoff errors accumulated in the recursion could destroy the accuracy of the result.

The memory consumption of the module is proportional to $l_{\max }^{2} m_{\max }$, and its runtime scales with $m_{\max } n_{\text {samples. }}$. Since interpolations for different pointings are independent, parallelisation was trivial to implement.

By default, the interpolator module calculates the total intensity observed by the detector. Optionally, any of the individual Stokes parameters $I, Q$, and $U$, can also be extracted, but this calculation is only exact for axisymmetric polarised beams.

When using linear interpolation, it has been observed that the power spectrum of the resulting co-added maps was suppressed in comparison to the input maps at high $l$. This is a consequence of the finite resolution of the ring set, combined with the non-perfect interpolation in $\vartheta$ and $\varphi$. Although this effect cannot be eliminated entirely, increasing the interpolation order and/or increasing $l_{\max , \text { out }}$ in the totalconcolver reduces the problem dramatically.

\subsubsection{Dipole}

This component calculates the time-dependent dipole signal observed by a given detector. The user can choose whether the kinematic dipole component (caused by the motion of the satellite in the Solar System, with the Solar System within the Galaxy, with the Galaxy within the Local Group, and so forth) should be included, whether relativistic effects should be calculated, whether only higher order corrections should be returned, etc. The default output quantity is the dipole signal in antenna temperature, but thermodynamic temperature can also be calculated.

\subsubsection{Point-source convolver}

The task of the point source convolver is to simulate time streams of the signal created by fixed and moving point sources. For fixed sources, this could also be achieved by adding another foreground to skymixer or almmixer, but this is not very practical because the maximum multipole moment required in the totalconvoler to accurately treat this kind of strongly localised sources would be very high. For moving sources, this solution is not viable at all, since all signal contributions entering the mixing modules must be constant over the mission time scale. 
For these reasons, the point source convolver operates in real space, taking the detector pointings as input and convolving the sources close to a given pointing with the properly oriented beam pattern. Obviously, an efficient retrieval of sources in the vicintity of the beam is crucial for the performance of the module. This was achieved by pre-sorting the point source catalog into a HEALPix map with low resulution $\left(N_{\text {side }}=16\right)$ and by identifying, for all detector pointings, the nearby point sources using the query_disc() function of HEALPix with a radius of several times the beam FWHM.

While fixed point sources stay in this HEALPix lookup table during a whole simulation run, the positions of the moving point sources are calculated once per pointing period. These positions are then sorted into the lookup table accordingly and taken out again after the signal for the pointing period has been calculated.

As mentioned above, it is recommended to choose a high value for the ringres parameter when calculating the point source signal, because of their very localised nature.

For the fixed point sources, the radiation flux in each Planck frequency band is taken from the point source cata$\log$. For moving point sources (i.e. planets and asteroids), the corresponding positions in time are calculated using the JPL HORIZONS system ${ }^{9}$, whereas the radiation intensity of the moving point sources is calculated using an extended Wright \& Odenwald thermal emission model (Wright 1976; Neugebauer et al. 1971), which has been adapted separately for rocky planets, gaseous planets, and asteroids and which accounts for effects like distance to the satellite, illumination geometry, beaming, surface roughness, heating and cooling of the surface, etc. This will be described in more detail in a separate publication (R. Hell, in preparation; see also Schäfer et al. 2004b).

\subsubsection{Sorption-cooler noise}

Temperature fluctuations in the detectors caused by the operation of Planck's sorption cooler constitute an important systematic effect and have to be included in realistic simulations of time-ordered data. To this end, the Planck simulation package contains the glissando code contributed by LFI, which models this effect and will be fully integrated with multimod in the future. Since the LFI and HFI detectors may react quite differently to temperature fluctuations, it is likely that a completely different code will ultimately be required for the HFI channels.

\subsubsection{Sampler}

The sampler component takes the idealised signal impinging on the detector as input, which is produced by the components described above, and applies to it a model of the detector's measurement process. Two effects play an important role:

- Every sample recorded by any detector is in reality the average of several fast samples taken in the time after the last slow sample.

\footnotetext{
${ }^{9}$ http://ssd.jpl.nasa.gov/horizons.html
}

- All HFI detectors have an exponential impulse response with a time constant $\tau_{\text {bol }}$, so that each fast sample must in turn be calculated via the integral

$$
s_{\text {bol }}(t)=\int_{-\infty}^{t} \mathrm{~d} t^{\prime} s_{\text {sky }}\left(t^{\prime}\right) \frac{\exp \left[-\left(t-t^{\prime}\right) / \tau_{\text {bol }}\right]}{\tau_{\text {bol }}} .
$$

The algorithm approximates this integral by taking $N$ unequally spaced samples:

$$
s_{\mathrm{bol}}(t) \approx \sum_{k=1}^{N} s_{\mathrm{sky}}\left(t_{k}^{\prime}\right),
$$

where $t_{k}^{\prime}=t+\tau_{\text {bol }} \ln (k / N)$.

A more detailed description of the implementation can be found in Grivell \& Mann (1999).

It should be mentioned that both effects introduce a time delay into the sampled time-ordered data, i.e. that the signal written with the time stamp $t_{n}$ was produced entirely from measurements at $t \leq t_{n}$, which in the case of HFI reflect a signal that was seen even earlier, because of the bolometer's time response. This must be taken into account when time-ordered signal data and detector pointings are used to produce sky maps.

The sampler code can be trivially parallelised and has perfect cache behaviour, so that it should scale very well on multiprocessor machines. As expected, the sampler module produces data at a rate equal to the detector's sampling frequency.

\subsection{7. $1 / f$-noise}

The electronics of both LFI and HFI detectors produces noise that has a power-law spectrum with spectral index $0 \leq \gamma \leq 2$ between a lower frequency $f_{\min }$ and a higher (or knee) frequency $f_{\text {knee }}$. Below and above those frequencies, the spectrum is white. The targeted spectral power is thus

$P_{n}^{*}(f)=P_{0}\left(1+\left(f / f_{\min }\right)^{\gamma}\right)^{-1}+P_{\text {white }}$,

where $P_{\text {white }}$ is the high-frequency, white-noise level, and $P_{0}=$ $\left.P_{\text {white }}\left(f_{\text {knee }} / f_{\min }\right)^{\gamma}-1\right)$. The value of $\gamma$ is $\approx 1.7$ for LFI and 2 for HFI detectors.

A noise time-stream with this spectrum could be obtained by modelling it in Fourier space with random coefficients and then performing an FFT. However, considering the fact that the coherence length for an HFI detector would be approximately $10^{8}$ samples, this method is rather expensive in terms of main memory consumption.

The noise is more efficiently generated by sampling and adding numerical solutions of stochastic differential equations (SDEs) of the form

$\dot{x}_{i}(t)=-x_{i}(t) / \tau_{i}+\xi_{i}(t)$,

where $\tau_{i}$ is the autocorrelation time of process $x_{i}(t)$. The $\xi_{i}(t)$ are individual white noise processes that are implemented in the discretised form of Eq. (10) as normalised series of independent Gaussian random numbers $\xi_{i}(t)$ with autocorrelation function $\left\langle\xi_{i}(t) \xi_{i}(s)\right\rangle=\delta(t-s)$. 
In order to have good logarithmic frequency coverage, a logarithmic grid of $\tau$ between $2 \pi / f_{\text {knee }}$ and $2 \pi / f_{\min }$ is used together with a process with $\tau_{0} \rightarrow 0$ for the high-frequency white noise $n_{0}(t)$.

The resulting SDE spectra

$P_{i}(f)=\left(\tau_{i}^{-2}+(2 \pi f)^{2}\right)^{-1}$

can be combined to an approximation of the target spectrum $P_{n}^{*}(f)$ (Eq. (9)) by combining the individual processes with properly chosen weights $c_{i}$ :

$n(t)=\sum_{i} c_{i} x_{i}(t)$,

$P_{n}(f)=\sum_{i} c_{i}^{2} P_{i}(f) \approx P_{n}^{*}(f)$.

More technical detail on choosing the weights, initialising the SDE processes, and optimising the computational performance will be covered in a separate publication (Enßlin et al., in preparation), but see also Keihänen et al. (2005).

Since the output of the noise generator is added to the output of the sampler module, both are naturally produced at the same sampling frequency.

Parallelisation of the algorithm is not straightforward, because every noise sample can only be computed after all of its predecessors have been calculated. This prohibits the most straightforward strategy of dividing the requested samples between multiple processors. In our approach, each SDE process calculates its associated time stream separately, which can be done in parallel processes, and all of these time streams are added at the end. While this solution can be significantly faster than a scalar implementation, its scalability is limited by the number of SDE processes $(\approx 10)$. Increasing the number of processors beyond this value achieves no further speedup.

\section{Summary and outlook}

We have described the current state of the Planck simulation pipeline in this paper. This package allows a complete simulation of the time-ordered data streams produced by the satellite's detectors, taking as input a set of cosmological parameters, various foreground emission models, a satellite scanning strategy, and parameters characterising the detectors and on-board electronics. The availability of such data sets allows extensive testing of the data analysis software for the mission already before launch, which greatly decreases the time interval between the availability of raw data and the publication of first results. Furthermore, the simulation modules can be conveniently used to study the influence of assumed systematic effects or changes in the mission parameters on the quality of the final data.

Obviously, many of the package's modules are still under development, and new modules are expected. Integration with the Planck Data Management Component and the mission's Integrated Data and Information System (IDIS) will be another important milestone. Any significant changes and enhancements will be presented in a follow-up paper.

The non-Planck-specific modules will hopefully be of use outside the Planck collaboration as well. This covers almost all of the presented codes, with the exception of the modules for the satellite dynamics and sorption-cooler noise. A future public release of the non-Planck-specific parts of the package is envisaged by the authors. Currently, a partial simulation pipeline, which is hosted by the German Astrophysical Virtual Observatory, can be tested interactively over the Internet ${ }^{10}$.

Acknowledgements. The work presented in this article was done in the course of the Planck satellite mission. The authors are grateful for many productive interactions with the Planck team.

We thank Mark Ashdown for implementation and support of the Beam package, Benjamin Wandelt for providing an initial implementation of the oofnoise and totalconvolver modules, Carlo Burigana, Davide Maino, and Krzysztof Górski for early FFT-based noise generation modules, Daniel Mortlock and Floor van Leeuwen for the simmission module, Ian Grivell and Bob Mann for the original sampler module, Aniello Mennella and Michele Maris for the glissando package, Fabrizio Villa and Maura Sandri for instrumental information on LFI, Bob Mann for compiling the first focal plane database and Mark Ashdown for maintaining it. Our thanks go to Giovanna Giardino, Bjørn Schäfer, Christoph Pfrommer, Nabila Aghanim, and François Bouchet for the contribution of template maps, Carlo Baccigalupi for contributing his dust emission model, and to Antony Lewis for helping with the integration of CAMB.

Some of the codes presented in this paper are based on the HEALPix package (Górski et al. 2005), the use of which is gratefully acknowledged.

\section{References}

Bluestein, L. I. 1968, Northeast Electronics Research and Engineering Meeting Record, 10, 218

Box, G. E. P., \& Muller, M. E. 1958, Ann. Math. Stat., 29, 610

Challinor, A., Fosalba, P., Mortlock, D., et al. 2000, Phys. Rev. D, 62, 123002

Challinor, A. D., Mortlock, D. J., van Leeuwen, F., et al. 2002, MNRAS, 331, 994

Colberg, J. M., White, S. D. M., Yoshida, N., et al. 2000, MNRAS, 319, 209

Dame, T. M., Hartmann, D., \& Thaddeus, P. 1996, BAAS, 28, 1362

Dame, T. M., Hartmann, D., \& Thaddeus, P. 2001, ApJ, 547, 792

Dolag, K., Hansen, F. K., Roncarelli, M., \& Moscardini, L. 2005, MNRAS, 363, 29

Finkbeiner, D. P. 2003, ApJS, 146, 407

Finkbeiner, D. P., Davis, M., \& Schlegel, D. J. 1999, ApJ, 524, 867

Frigo, M., \& Johnson, S. G. 1998, in Proc. 1998 IEEE Intl. Conf. Acoustics Speech and Signal Processing, 3 (IEEE), 1381

Górski, K. M., Banday, A. J., Hivon, E., \& Wandelt, B. D. 2002, in Astronomical Data Analysis Software and Systems XI, ASP Conf. Ser., 281, 107

Górski, K. M., Hivon, E., Banday, A. J., et al. 2005, ApJ, 622,759

Giardino, G., Banday, A. J., Górski, K. M., et al. 2002, A\&A, 387, 82

Grivell, I., \& Mann, R. 1999, Sampler module for scan strategy simulations, Planck LiveLink repository

Jenkins, A., Frenk, C. S., White, S. D. M., et al. 2001, MNRAS, 321, 372

Keihänen, E., Kurki-Suonio, H., \& Poutanen, T. 2005, MNRAS, 360, 390

Lewis, A., Challinor, A., \& Lasenby, A. 2000, ApJ, 538, 473

Marsaglia, G. 2003, J. Statistical Software, 8

\footnotetext{
${ }^{10}$ http://www.g-vo.org/portal/tile/products/services/ planck/index.jsp
} 
Neugebauer, G., Miinch, G., Kieffer, H., Chase, S. C., \& Miner, E. 1971, AJ, 76, 719

NOST. 1999, Definition of the Flexible Image Transport System (FITS), http://archive.stsci.edu/fits/fits_standard/

Pasian, F., \& Sygnet, J. 2002, in Astronomical Data Analysis II. ed. J.-L. Starck, \& F. D. Murtagh, Proc. SPIE, 4847, 25

Pence, W. 1999, in Astronomical Data Analysis Software and Systems VIII, ASP Conf. Ser., 172, 487

Schäfer, B. M., Pfrommer, C., Bartelmann, M., Springel, V., \& Hernquist, L. 2004a, MNRAS, submitted [arXiv: astro-ph/0407089]

Schäfer, B. M., Pfrommer, C., Hell, R., \& Bartelmann, M. 2004b, MNRAS, submitted [arXiv:astro-ph/0407090]
Schlegel, D. J., Finkbeiner, D. P., \& Davis, M. 1998, ApJ, 500, 525

Seljak, U., \& Zaldarriaga, M. 1996, ApJ, 469, 437

Swarztrauber, P. 1982, Vectorizing the Fast Fourier Transforms (New York: Academic Press), 51

Taffoni, G., Maino, D., Vuerli, C., et al. 2005, IEEE Transactions on Computers, in press

Tauber, J. A. 2004, Adv. Space Res., 34, 491

Valls-Gabaud, D. 1998, PASA, 15, 111

van Leeuwen, F., Challinor, A. D., Mortlock, D. J., et al. 2002, MNRAS, 331, 975

Wandelt, B. D., \& Górski, K. M. 2001, Phys. Rev. D, 63, 123002

Wright, E. L. 1976, ApJ, 210, 250

Zaldarriaga, M., \& Seljak, U. 1997, Phys. Rev. D, 55, 1830 